\title{
A review of unusual species of Cotesia (Hymenoptera, Braconidae, Microgastrinae) with the first tergite narrowing at midlength
}

\author{
Ankita Gupta', Mark Shaw ${ }^{2}$, Sophie Cardinal ${ }^{3}$, Jose Fernandez-Triana ${ }^{3}$ \\ I ICAR-National Bureau of Agricultural Insect Resources, P. B. No. 2491, H. A. Farm Post, Bellary Road, \\ Hebbal, Bangalore, 560 024, India 2 National Museums of Scotland, Edinburgh, United Kingdom 3 Canadian \\ National Collection of Insects, Ottawa, Canada \\ Corresponding author: Ankita Gupta (drankitagupta7@gmail.com) \\ Academic editor: K. van Achterberg | Received 9 February 2016 | Accepted 14 March 2016 | Published 12 April 2016 \\ http://zoobank.org/9EBC59EC-3361-4DD0-A5A1-D563B2DE2DF9 \\ Citation: Gupta A, Shaw M, Cardinal S, Fernandez-Triana J (2016) A review of unusual species of Cotesia \\ (Hymenoptera, Braconidae, Microgastrinae) with the first tergite narrowing at midlength. ZooKeys 580: 29-44. doi: \\ $10.3897 /$ zookeys. 580.8090
}

\begin{abstract}
The unusual species of Cotesia (Hymenoptera, Braconidae, Microgastrinae) with the first tergite narrowing at midlength are reviewed. One new species, Cotesia trabalae sp. n. is described from India and compared with Cotesia pistrinariae (Wilkinson) from Africa, the only other species sharing the same character of all the described species worldwide. The generic placement of these two species, based on molecular and morphological analyses as well as parasitoid biology is discussed.
\end{abstract}

\section{Keywords}

Cotesia trabalae, new species, Trabala vishnou, India, Cotesia pistrinariae, Mylothris chloris, Africa

\section{Introduction}

With 269 described species (Fernandez-Triana and Ward 2015), Cotesia is the second largest genus in the hyperdiverse subfamily Microgastrinae (Hymenoptera, Braconidae), with estimates of its actual diversity ranging from 1,500 (Mason 1981) to 2,500 species (van Achterberg and Polaszek 1996). The genus was described as monotypic by

Copyright Ankita Gupta et al. This is an open access article distributed under the terms of the Creative Commons Attribution License (CC BY 4.0), which permits unrestricted use, distribution, and reproduction in any medium, provided the original author and source are credited. 
Cameron in 1891, but soon after it was synonymized under Apanteles (Szépligeti 1904: 105) and remained that way until Mason (1981) reinstated it as valid and transferred a number of species to it. Mason estimated that $30-40 \%$ of the temperate species previously considered as 'Apanteles' actually belong to Cotesia, while in tropical areas that proportion is only 10-20\% (Mason 1981: 113). Austin and Dangerfield (1992: 21) considered Cotesia to be the largest genus within Microgastrinae - although that assumption is questionable, based on described and undescribed species available in collections it is clear that Apanteles and Glyptapanteles are much more diverse, especially in the tropics. Regardless, Cotesia comprises a huge assemblage of species and it is found in all biogeographical regions of the planet (Yu et al. 2012).

In spite of its diversity, species of Cotesia tend to be relatively uniform morphologically, especially regarding the shape of tergites 1-3 and propodeum sculpture. When redescribing the genus, Mason (1981: 110-111) stated '...Tergite I occasionally wider than long but usually a little longer than wide and broadened apically, occasionally somewhat barrel-shaped or parallel-sided, but never narrowed apically; never with a median apical depression... Tergite I frequently smooth basally but the posterior part almost invariably rugose or rugopunctate... Propodeum invariably rugose and never with an areolet; usually with a median longitudinal carina that may be partially obscured by rugosity and usually an incomplete transverse carina laterally separating the rugose declivity from a smoother anterior area...'

Until now only one species of Cotesia was known to have a significantly different shape of mediotergite 1 (henceforward abbreviated as T1). The species Cotesia pistrinariae (Wilkinson, 1929) has T1 strongly narrowing at midlength so that T1 width medially (at narrowest point) is $0.5-0.6 \times$ its width at anterior margin and $0.6-0.7 \times$ its width at posterior margin. The shape of $\mathrm{T} 1$ was so bizarre that in the original description of the species, as 'Apanteles pistrinariae', Wilkinson (1929: 445) wrote '... The unusual form of the 1st tergite, although a character whereby the species may be immediately separated from all others, renders the satisfactory placing of this species in my key a difficult matter...' Even at that time, when species of Cotesia were still considered to be part of a much expanded 'Apanteles' genus, this species was hard to place within a group.

In our studies of the world fauna of Microgastrinae we have found a new species of Cotesia with similar shape of T1 (narrowing at midlength), which is described below, together with diagnostic characters to separate it from $C$. pistrinariae. We discuss further the generic placement of those two species, based on molecular and morphological analyses as well as parasitoid biology.

\section{Methods}

This paper is based on study of Cotesia specimens collected in India (housed at the ICARNational Bureau of Agricultural Insect Resources (NBAIR), Bangalore, India); and Africa (housed in the National Museums of Scotland (NMS), Edinburgh, United Kingdom). 
Morphological terms and measurements of structures are mostly as in Mason (1981), Huber and Sharkey (1993), and Whitfield (1997).

Photos of the Indian species were taken with a Leica M 205 A stereozoom microscope with Leica DC 420 inbuilt camera using automontage software (version 3.8). Photos of the African species were taken with a Keyence VHX-1000 Digital Microscope, using a lens with a range of 13-130 $\times$. Multiple images through the focal plane were taken of a structure and these were combined to produce a single in-focus image using software associated with the Keyence System.

DNA barcodes were obtained using DNA extracts prepared from single legs using a glass fibre protocol (Ivanova et al. 2006). Briefly, total genomic DNA was re-suspended in $30 \mu \mathrm{l}$ of $\mathrm{dH} 2 \mathrm{O}$, and the standard barcoding region near the 5' terminus of the COI gene was amplified using standard primers (LepF1- LepR1) following established protocols (Smith et al. 2006, 2007, 2008). If the initial amplification was unsuccessful, shorter sequences were generated using internal primers and subsequently contigued together. A MUSCLE sequence alignment was generated in Geneious 8.1.7 (http:// www.geneious.com, Kearse et al. 2012) for 241 species of Cotesia with sequences over 600 base pairs available in the Barcode of Life Data System (BOLD, http://www. boldsystems.org/) (Ratnasingham and Hebert 2007). In addition, sequences from species of most other genera of Microgastrinae were used as outgroups when available (120 outgroup sequences). All information for individual specimens in BOLD can be retrieved by Process ID (sequence accession) or Sample ID (voucher codes); and the newly described species can be retrieved from Genbank (codes KJ459172, KJ459169, KM875666, KT308157 and KT308158) (information summarized in Table 1 for the new species).

Both ends of the sequence alignment were trimmed to reduce missing data and a neighbor-joining tree based on Kimura 2-parameter distances was generated in Geneious 8.1.7. A Bayesian majority rule consensus tree was generated in MrBayes 3.2.1 (Ronquist et al. 2011). To find the best-fit partitioning scheme and models of molecular evolution for the nucleotide alignment, PartitionFinder v1.1.1 (Lanfear et al. 2012) was used. Two independent runs of 20 million generations in which each codon position formed a partition with a GTR $+\mathrm{IG}$ model applied (based on results of PartitionFinder analysis) were analysed. To ensure that both runs had converged and reached stationarity, trace files of all estimated parameters were observed and the estimated sample size of each parameter was verified to be over 200 . The first $10 \%$ of samples were removed as burn-in.

\section{Results}

To date, only two species of Cotesia are known to have a T1 narrowing at midlength. That represents less than $1 \%$ of all described species worldwide. In the neighbor-joining tree both species cluster more closely with other species (Fig. 1), and in the Bayesian tree (Fig. 2) they are part of a large unresolved polytomy which provides no support for them being 


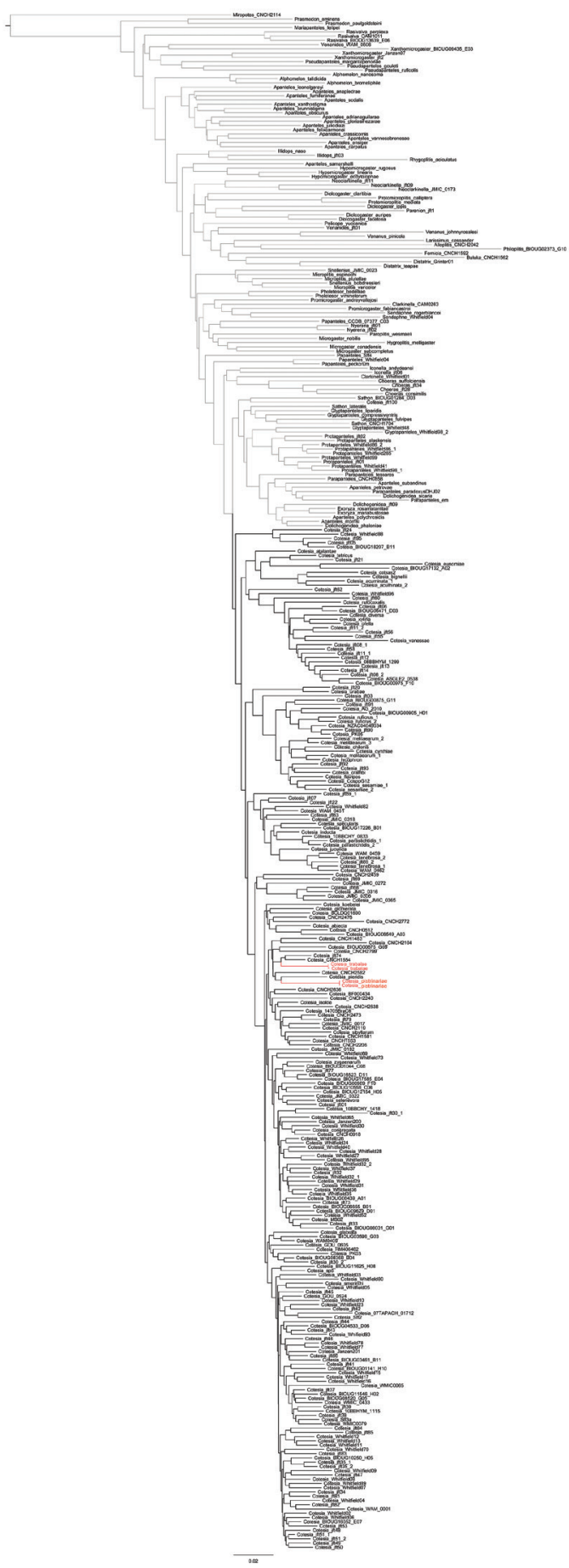

Figure I. Neighbor-joining tree based on Kimura 2-parameter distances of 241 species of Cotesia and 120 species of other genera of Microgastrinae. The two Cotesia species known to have T1 narrowing at midlength are colored in red. 


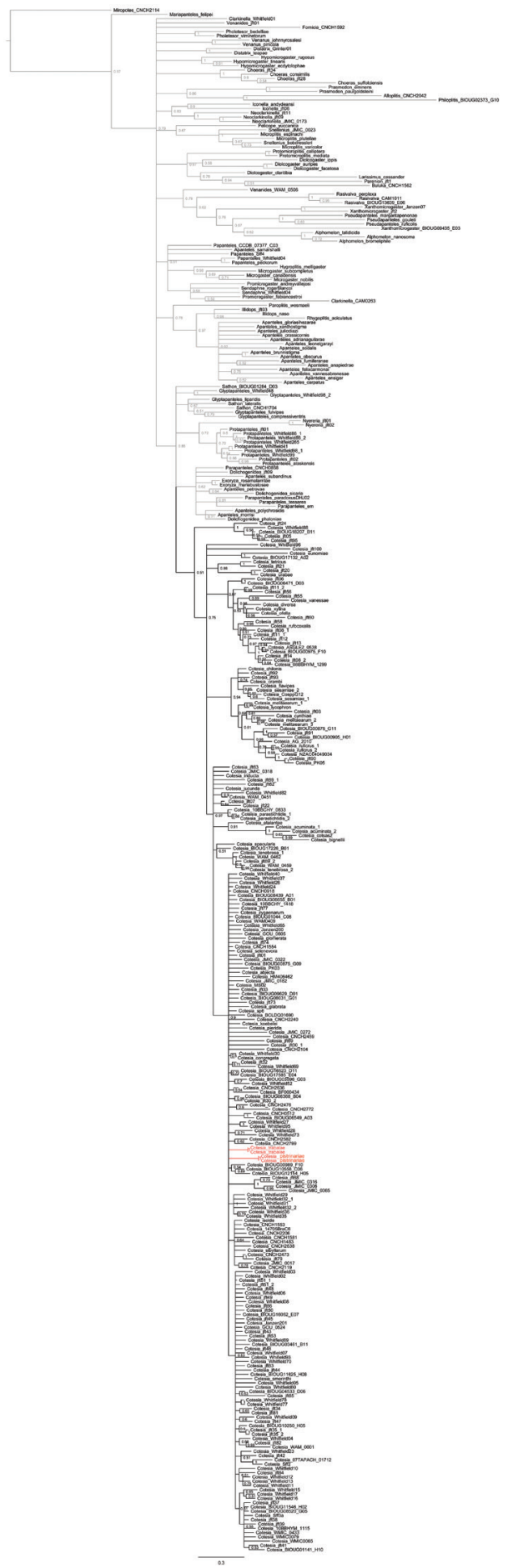

Figure 2. Bayesian majority rule concensus tree of 241 species of Cotesia and 120 species of other genera of Microgastrinae. The two Cotesia species known to have T1 narrowing at midlength are colored in red. 
Table I. Showing comparative measurements from different localities.

\begin{tabular}{|c|c|c|c|}
\hline Female characters & Kasaragod & Shimla & Meghalaya \\
\hline Body length in $\mathrm{mm}$ & $2.59,2.51,2.48,2.44,2.58,2.60$ & $2.62,2.66,2.43$ & 2.73 \\
\hline Fore wing length & $\begin{array}{l}2.42 \text { (for body length } 2.59 \mathrm{~mm} \text { ) } \\
2.23 \text { (for body length } 2.51 \mathrm{~mm} \text { ) }\end{array}$ & $\begin{array}{c}2.59 \text { (for body } \\
\text { length } 2.62 \mathrm{~mm} \text { ) }\end{array}$ & 2.70 \\
\hline Antenna length/body length & $\begin{array}{l}2.546 \text { (for body length } 2.59 \mathrm{~mm} \text { ) } \\
2.438 \text { (for body length } 2.51 \mathrm{~mm} \text { ) }\end{array}$ & \begin{tabular}{|c|}
2.52 (for body \\
length $2.62 \mathrm{~mm}$ ) \\
\end{tabular} & \\
\hline $\begin{array}{l}\text { Ratio of ocular-ocellar line/posterior } \\
\text { ocellus diameter }\end{array}$ & 1.50 & $2.00-2.03$ & 1.89 \\
\hline $\begin{array}{l}\text { Ratio of interocellar distance/posterior } \\
\text { ocellus diameter }\end{array}$ & 1.83 & $1.98-2.02$ & 2.23 \\
\hline $\begin{array}{c}\text { Antennal flagellomere } 2 \\
\text { (ratio of length/width) }\end{array}$ & 3.50 & $2.36-3.05$ & 2.73 \\
\hline $\begin{array}{l}\text { Antennal flagellomere } 14 \\
\text { (ratio of length/width) }\end{array}$ & 1.83 & $1.91-1.92$ & 1.92 \\
\hline $\begin{array}{l}\text { Ratio of length of flagellomere } \\
\text { 2/length of flagellomere } 14\end{array}$ & 1.91 & $1.83-1.85$ & 1.59 \\
\hline Ratio of metafemur length/width & 3.47 & $3.57-4.20$ & 3.30 \\
\hline Number of pits in scutoscutellar sulcus & 9 & 9 & 9 \\
\hline $\begin{array}{l}\text { Ratio of mediotergite } 1 \text { width at anterior } \\
\text { margin/width at posterior margin: }\end{array}$ & $1.01-1.05$ & $0.77-0.78$ & 0.88 \\
\hline $\begin{array}{l}\text { Ratio of mediotergite } 1 \text { median } \\
\text { width/ width at posterior margin }\end{array}$ & $0.86-0.92$ & $0.77-0.81$ & 0.83 \\
\hline $\begin{array}{l}\text { Ratio of mediotergite } 2 \text { width at } \\
\text { posterior margin/length }\end{array}$ & $2.87-2.94$ & $2.26-2.32$ & 2.32 \\
\hline $\begin{array}{l}\text { Ratio of ovipositor sheaths } \\
\text { length/metatibial length }\end{array}$ & 0.16 & 0.21 & $0.16-0.17$ \\
\hline $\begin{array}{l}\text { Ratio of metatibia inner spur } \\
\text { length/metabasitarsus length }\end{array}$ & 0.66 & $0.57-0.59$ & 0.61 \\
\hline $\begin{array}{l}\text { Ratio of maximum height of } \\
\text { mesoscutellum lunules/maximum height } \\
\text { of lateral face of mesoscutellum }\end{array}$ & 0.30 & 0.45 & 0.37 \\
\hline Ratio of length of fore wing veins $r / 2 R S$ & 1.22 & $0.75-0.77$ & 1.06 \\
\hline $\begin{array}{l}\text { Ratio of length of fore wing } \\
\text { veins } 2 \mathrm{RS} / 2 \mathrm{M} \text { : }\end{array}$ & 1.44 & $1.64-1.78$ & 1.73 \\
\hline $\begin{array}{l}\text { Ratio of length of fore wing veins } 2 \mathrm{M} / \\
(\mathrm{RS}+\mathrm{M}) \mathrm{b}\end{array}$ & 1.25 & $1.25-1.38$ & 1.25 \\
\hline Pterostigma (ratio of length/width) & 2.53 & $2.52-3.00$ & 2.55 \\
\hline $\begin{array}{l}\text { Ratio of lengths: meta basitarsus/inner } \\
\text { metatibial spur/outer metatibial spur }\end{array}$ & $0.35 / 0.23 / 0.15$ & $\begin{array}{l}0.37 / 0.21 / 0.14 \\
0.37 / 0.22 / 0.14 \\
\end{array}$ & $0.43 / 0.26 / 0.15$ \\
\hline
\end{tabular}

sister species, although it does not preclude that possibility either. However, the molecular data support the monophyly of Cotesia, including both C. pistrinariae and C. trabalae.

Cotesia pistrinariae, from Africa (Fig. 3), has the propodeum with transverse and median carinae weakly defined and only partially visible, and without traces of lateral carinae or areola. The hypopygium is relatively large (clearly protruding beyond apex of metasoma) and with numerous and long setae. T1 is narrower: at approximately half its length it is $0.5-0.6 \times$ as wide as its width at the anterior margin and 0.6-0.7 $\times$ 

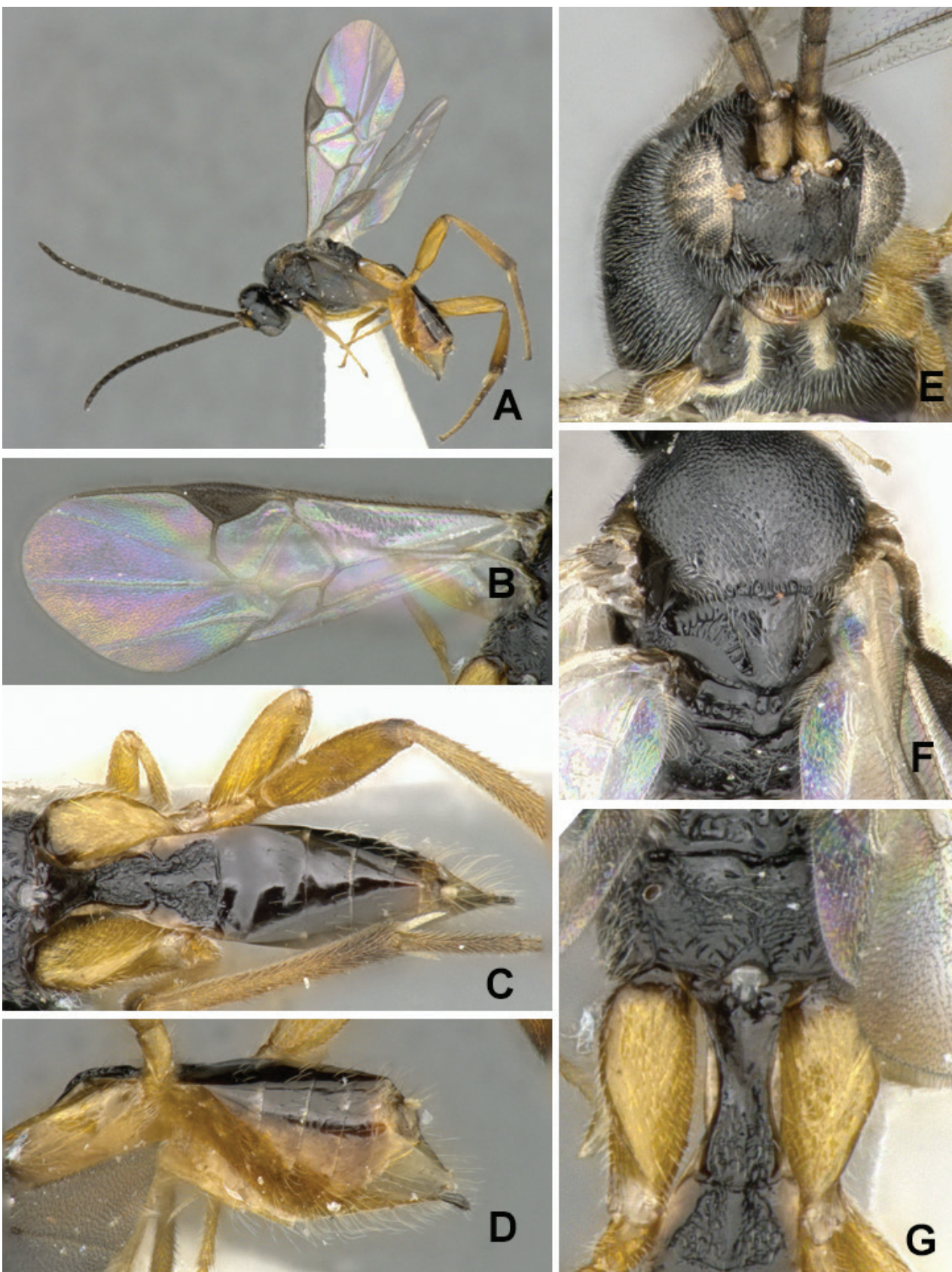

Figure 3. Cotesia pistrinariae. A Habitus, lateral view B Fore wing C Metasoma, dorsal view D Metasoma lateral view $\mathbf{E}$ Head, frontal view $\mathbf{F}$ Mesosoma, dorsal view $\mathbf{G}$ Details of propodeum, T1 and T2, dorsal view. 
its width at the posterior margin of the tergite. The species is rather widely distributed in Africa (Cape Verde Islands, Democratic Republic of Congo, Eritrea, Ethiopia, Malawi, Nigeria, Rwanda, South Africa). We observed slight differences in coloration among specimens from different countries, but could not find any evidence to separate them and thus all are considered here to belong to the same species -although future studies might change that. All known caterpillar hosts belong to the family Pieridae (Mylothris chloris (Fabricius) and undetermined gregariously feeding species).

The Indian species, Cotesia trabalae sp. n., described below, is obviously different (Figs 4-6). The propodeum has transverse and median carinae which are clearly defined and complete, as well as two partial lateral carinae on the posterior half of the propodeum (which seem to define a partial areola). The hypopygium is relatively small (not protruding beyond apex of metasoma) and mostly without setae. T1 is wider than in $C$. pistrinariae; its narrowest width, at approximately the half length of the tergite, is $0.8 \times$ (rarely up to 0.9 $x$ ) its width at the anterior and posterior margins of the tergite. The species is known only from India. The caterpillar hosts belong to Lasiocampidae (Trabala vishnou (Lefebvre)).

The carination pattern on the propodeum of C. trabalae is rather unusual. According to Mason's definition of the genus, Cotesia never has an areola on the propodeum (Mason 1981: 111), although this could be argued against, as certain species currently included in the genus seem to have a similar carination pattern to that found in C. trabalae [see, for example, illustrations of the propodeum for the species Cotesia rubripes (Haliday, 1834) and C. lineola (Curtis, 1830), as detailed by Wilkinson (1945, figs 27 and 57 in that paper)].

The definition and limits of the genus Cotesia are beyond the scope of this paper and will require a comprehensive study of the world fauna - including closely related genera such as Protapanteles. But for the time being we are considering all of the species dealt with in this paper as belonging to Cotesia based on the available evidence. In spite of the unique shape of T1 (and the rather unusual carination pattern of the propodeum in C. trabalae), the rest of the morphological characters analyzed strongly suggest that those two species are best placed in Cotesia. The molecular data also support the monophyly of the genus (Figs 1,2).

\section{Cotesia trabalae Gupta, sp. n.}

http://zoobank.org/6DEF35EA-D67F-4AF7-B50F-1ABF5A4FF28C

Figs 4-6

Type material. Holotype $q$ (NBAIR), INDIA, Kerala, Kasaragod, $12.5013^{\circ} \mathrm{N}$; 74.9900 E, 10.xii.2013, ex: caterpillar of Trabala vishnou (Lefebvre), NBAIR, Code 101213, DNA Voucher-BR-2014 (NBAIR).

Specimens examined. Paratypes: 5 (NBAIR) [part of the same brood as holotype]; 5 \& (NBAIR), INDIA: Himachal Pradesh, Shimla, 30.viii.2014, ex: caterpillar of Trabala vishnou (Lefèbvre) on Rubus sp.; 3 (NBAIR), INDIA: Meghalaya, Barapani, 25.x.2014, ex: caterpillar of Trabala vishnou (Lefèbvre) on Ricinis communis L. 

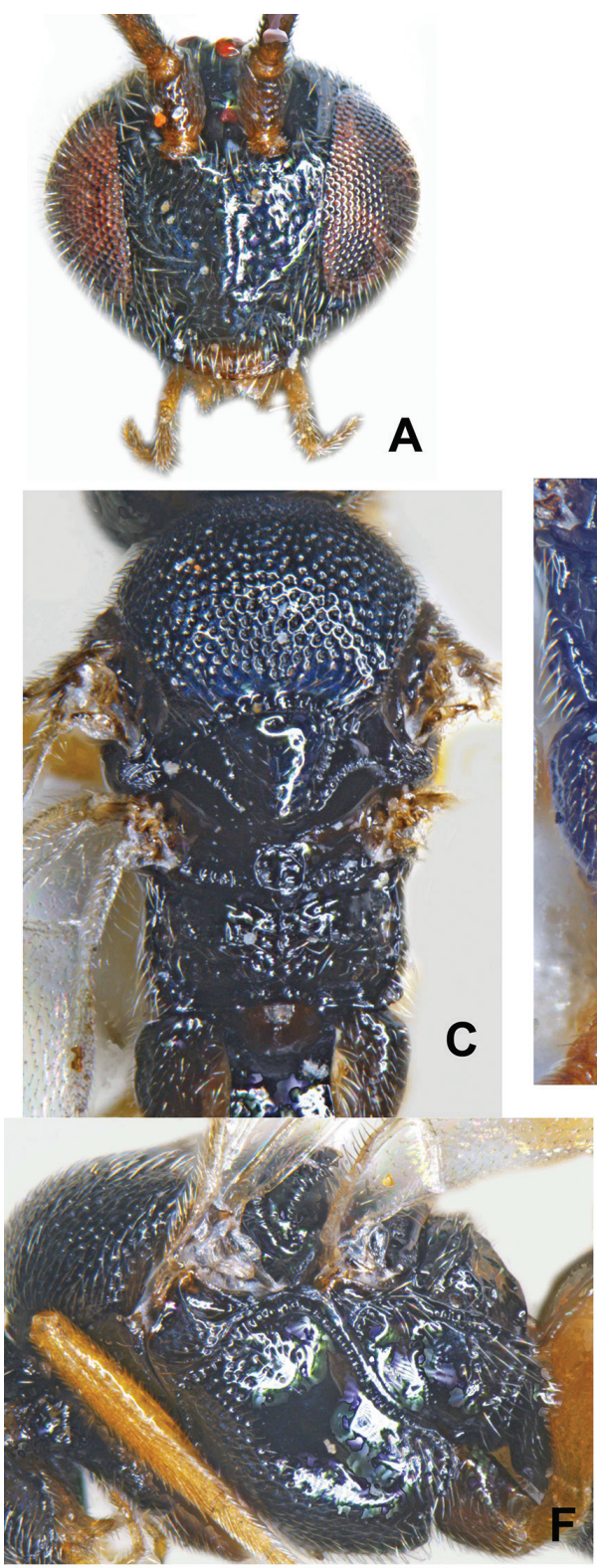
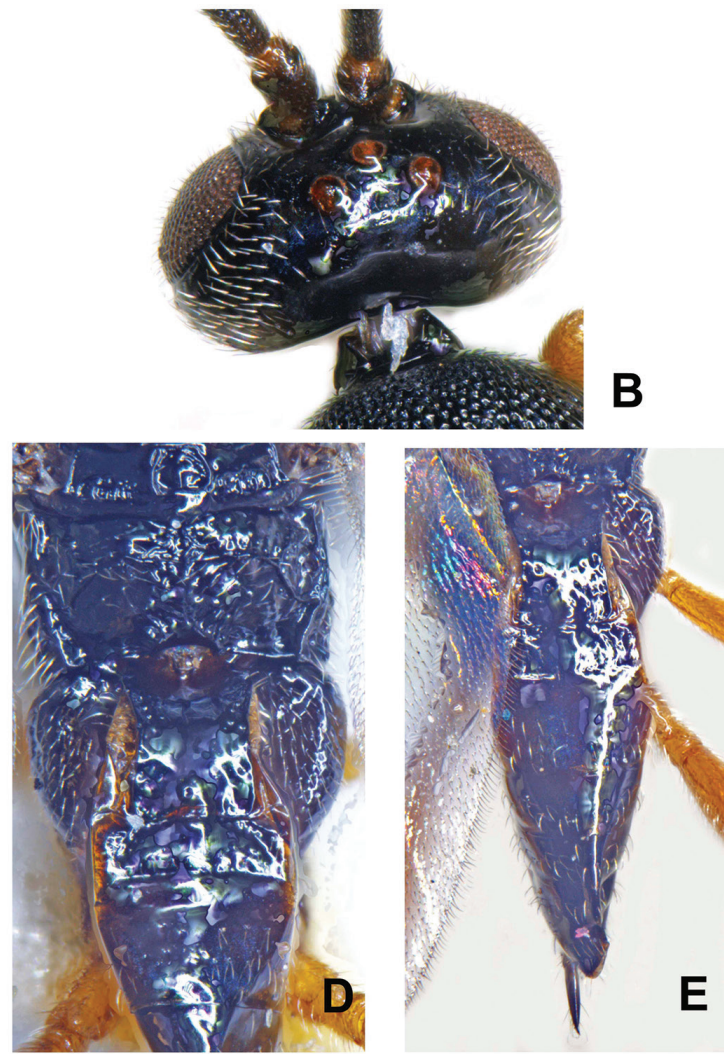

E

Figure 4. Cotesia trabalae sp. n. (Kasaragod): A Head in frontal view B Vertex C Mesosoma with propodeum in part $\mathbf{D}$ Propodeum with metasoma in part $\mathbf{E}$ Metasoma $\mathbf{F}$ Mesopleuron $\mathbf{G}$ Metasoma in lateral view.

Description. Female (Figs 5A, 6A). Body in lateral view: not distinctly flattened dorso-ventrally. Body length (head to apex of metasoma): 2.43-2.66 mm to $2.73 \mathrm{~mm}$. Fore wing: length: 2.42 (for body length $2.59 \mathrm{~mm}$ ), 2.23 (for body length $2.51 \mathrm{~mm})$. 

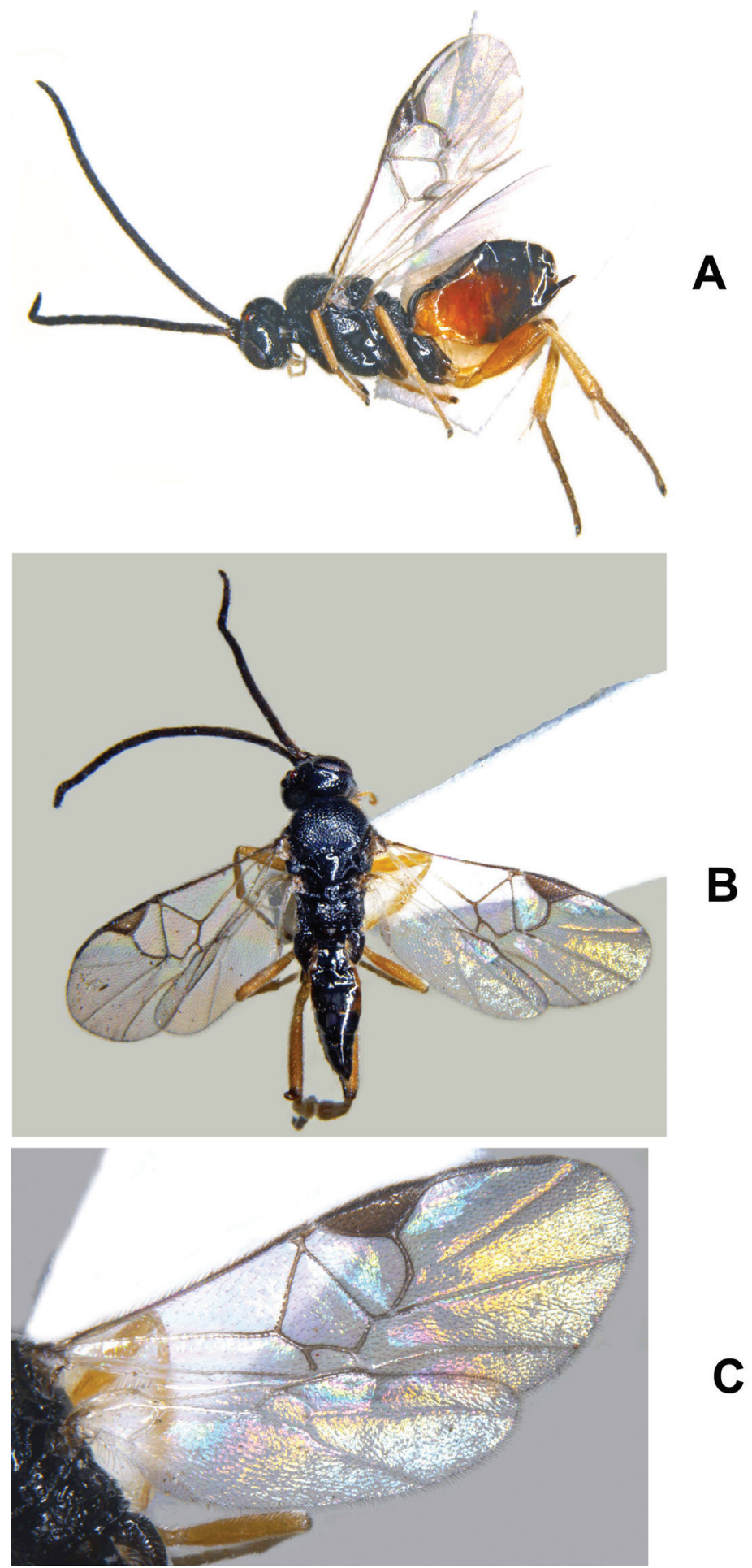

Figure 5. Cotesia trabalae sp. n. (Kasaragod): A Female in habitus B Female in dorsal view C Wings. 

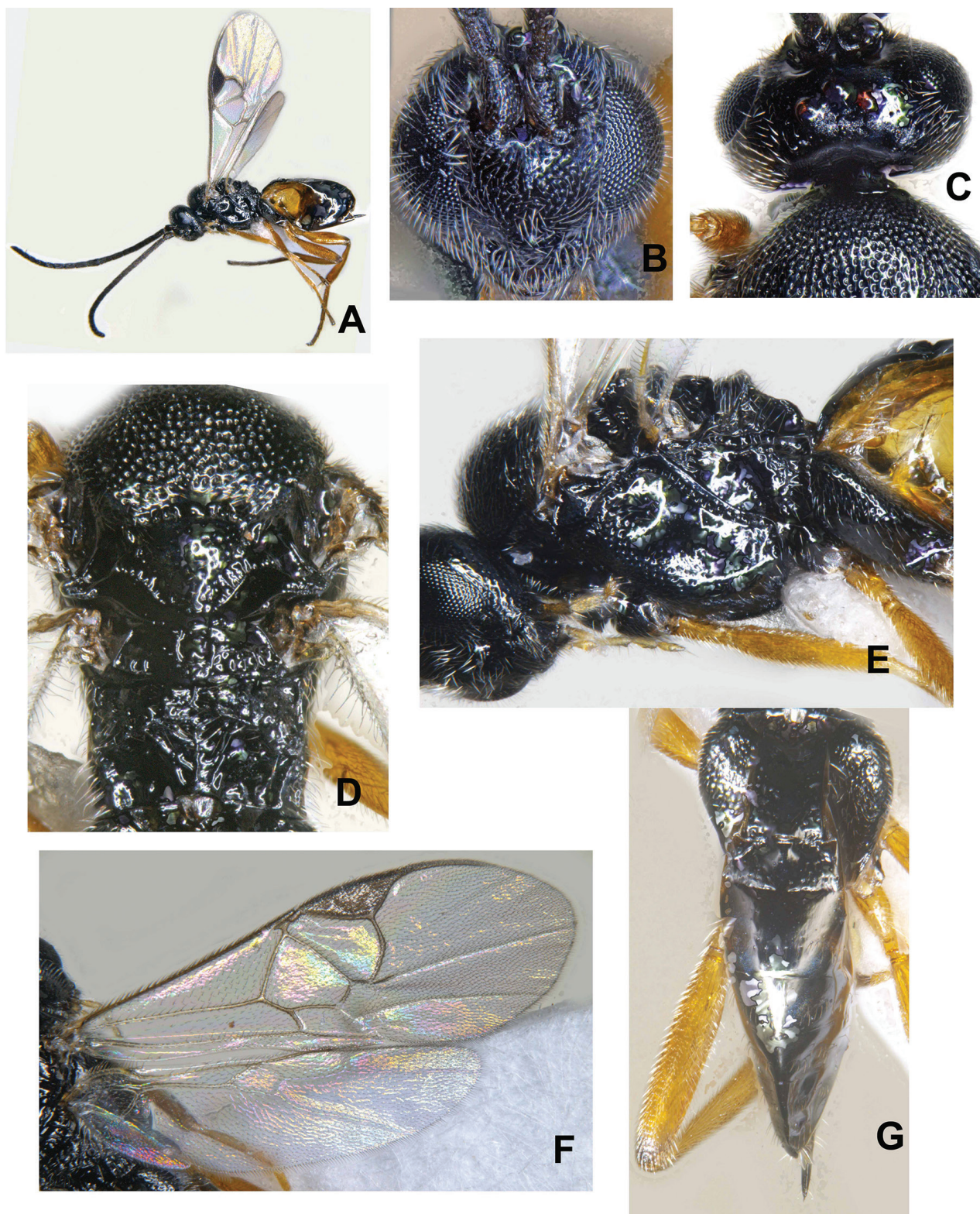

Figure 6. Cotesia trabalae sp. n. (Shimla): A Female in habitus B Head in frontal view C Vertex D Mesosoma with propodeum in part $\mathbf{E}$ Mesopleuron $\mathbf{F}$ Wings $\mathbf{G}$ Metasoma

Color. Body mostly black except for yellowish brown sternites in anterior half. Antenna color: scape, pedicel, and flagellum dark. Pro- and meso- coxae color: brown. Meta- coxa color: black. Pro- and meso- femur color: yellow. Meta- femur color: yellow, except for dark brown coloration on extreme apical tip. Metatibia and metatarsus color: yellowish brown. Tegula and humeral complex color: dark brown. Pterostig- 
ma color: mostly brown. Fore wing veins color: partially pigmented (r, RS, $2 \mathrm{M}$ and $(\mathrm{RS}+\mathrm{M}) \mathrm{b}$ dark; remaining pale).

Head. Antenna length/body length: antenna 0.96-0.98 $\times$ as long as body (head to apex of metasoma). Ocular-ocellar line/posterior ocellus diameter: 1.5-2.03. Interocellar distance/posterior ocellus diameter: 1.82-2.23. Antennal flagellomere 2 length/ width: 2.36-3.5. Antennal flagellomere 14 length/width: 1.83-1.91. Length of flagellomere 2/length of flagellomere 14: 1.59-1.9. Tarsal claws: simple. Metafemur length/ width: 3.3-4.2. Metatibia inner spur length/metabasitarsus length: 0.57-0.66.

Mesosoma. Anteromesoscutum: mostly with deep, dense punctures (separated by less than $2.0 \times$ their maximum diameter). Mesoscutellar disc: with shallow punctures scattered all over. Number of pits in scutoscutellar sulcus: 9. Maximum height of mesoscutellum lunules/maximum height of lateral face of mesoscutellum: 0.3-0.45. Propodeum: with prominent median carina, including transverse carina extending to spiracle; as well as two partial lateral carinae on the posterior half of the propodeum (which seem to define a partial areola). Sculpture: anterior 0.3 strongly rugose (carinae mostly radiating from strong longitudinal median carina), smooth and shiny, costula present.

Wings. Length of fore wing veins r/2RS: 0.75-1.22. Length of fore wing veins 2RS/2M: 1.44-1.78. Length of fore wing veins $2 \mathrm{M} /(\mathrm{RS}+\mathrm{M}) \mathrm{b}: 1.25-1.38$. Pterostigma length/width: 2.52-3.0. Point of insertion of vein $r$ in pterostigma: clearly beyond half length of pterostigma. Angle of vein $r$ with fore wing anterior margin: clearly outwards, inclined towards fore wing apex. Shape of junction of veins $r$ and $2 R S$ in fore wing: distinctly angled.

Metasoma. Mediotergite 1 shape: parallel-sided anteriorly, narrowing at midlength, slightly widened posteriorly. Mediotergite 1 width at anterior margin/width at posterior margin: $0.77-0.88$. Mediotergite 1 sculpture: smooth and shiny, except for widely scattered puncture at lateral margin and more so in the posterior half. Mediotergite 2 width at posterior margin/length: 2.26-2.94. Mediotergite 2 sculpture: mostly smooth. Outer margin of hypopygium: wide, semi-transparent. Ovipositor thickness: slightly tapering apically. Ovipositor sheaths length/metatibial length: 0.16-0.17, rarely 0.21.

Male. As female.

Molecular data. GenBank Accession numbers: KM875666, KT308157 and KT308158.

Distribution. India: Himachal Pradesh (Shimla), Kerala (Kasaragod), and Meghalaya (Barapani).

Biology/ecology. Host (Fig. 7): Trabala vishnou (Lefèbvre) (Lasiocampidae) on Ricinis communis L. (in Meghalaya), Rubus sp. (in Shimla), and one indeterminate wild plant in southern India (in Kerala).

Etymology. The name refers to the host species.

Comments. General body coloration remains the same for all the populations, however minor variations were noticed: (i) south Indian population (from Kasaragod) has comparatively lesser ratio of ocular-ocellar line/posterior ocellus diameter: 1.50 vs 1.89-2.03 in both north Indian populations; (ii) ratio of mediotergite 1 width at 

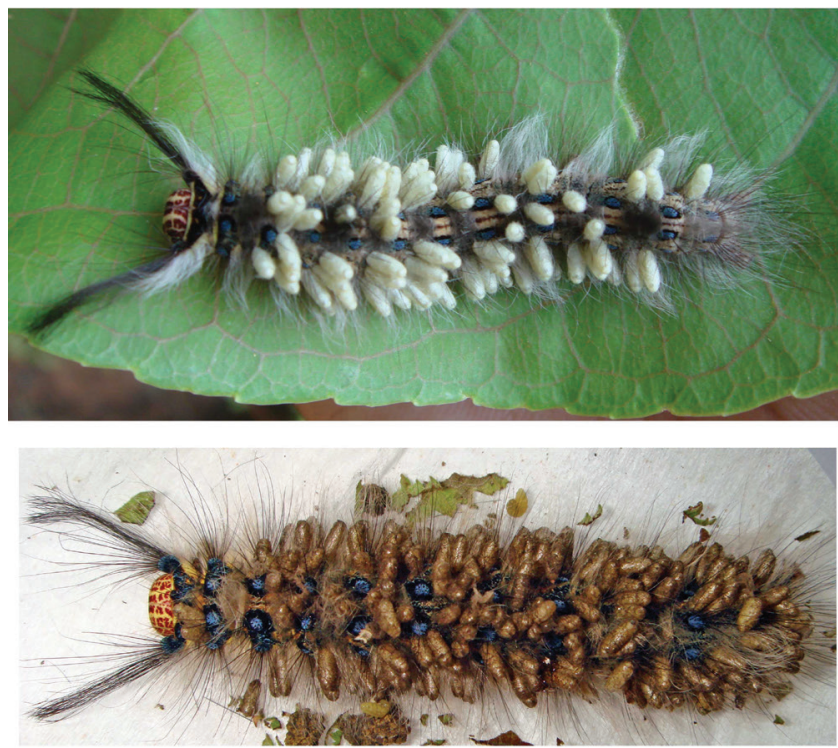

B

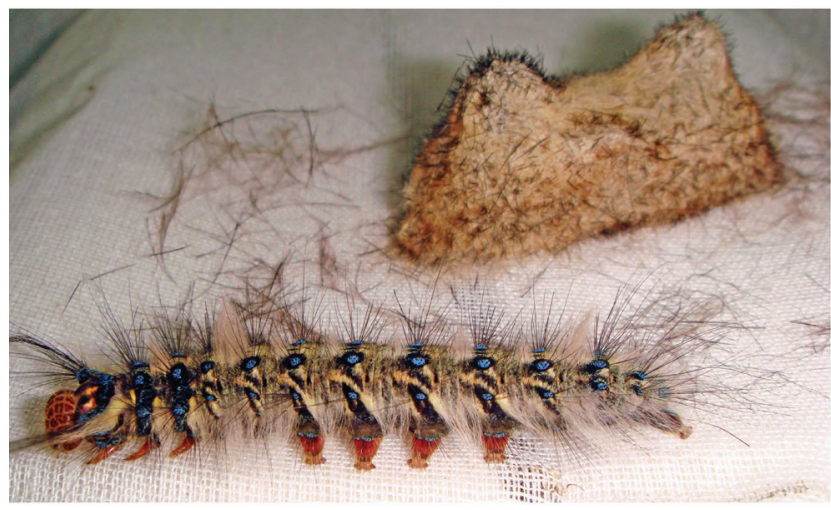

C

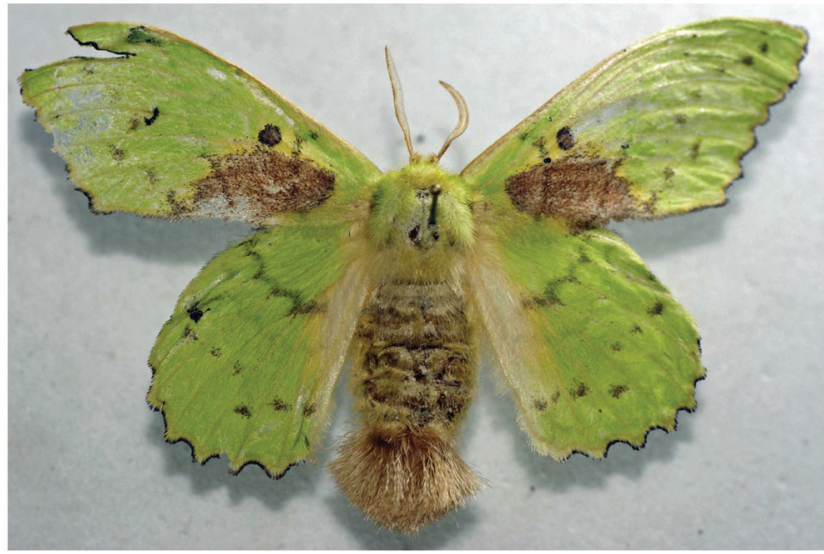

D

Figure 7. Trabala vishnou (Lefebvre): A Parasitized caterpillar (Kasaragod) B Parasitized caterpillar (Shimla) C Unparasitized caterpillar and cocoon (Shimla) D Adult (Shimla). 


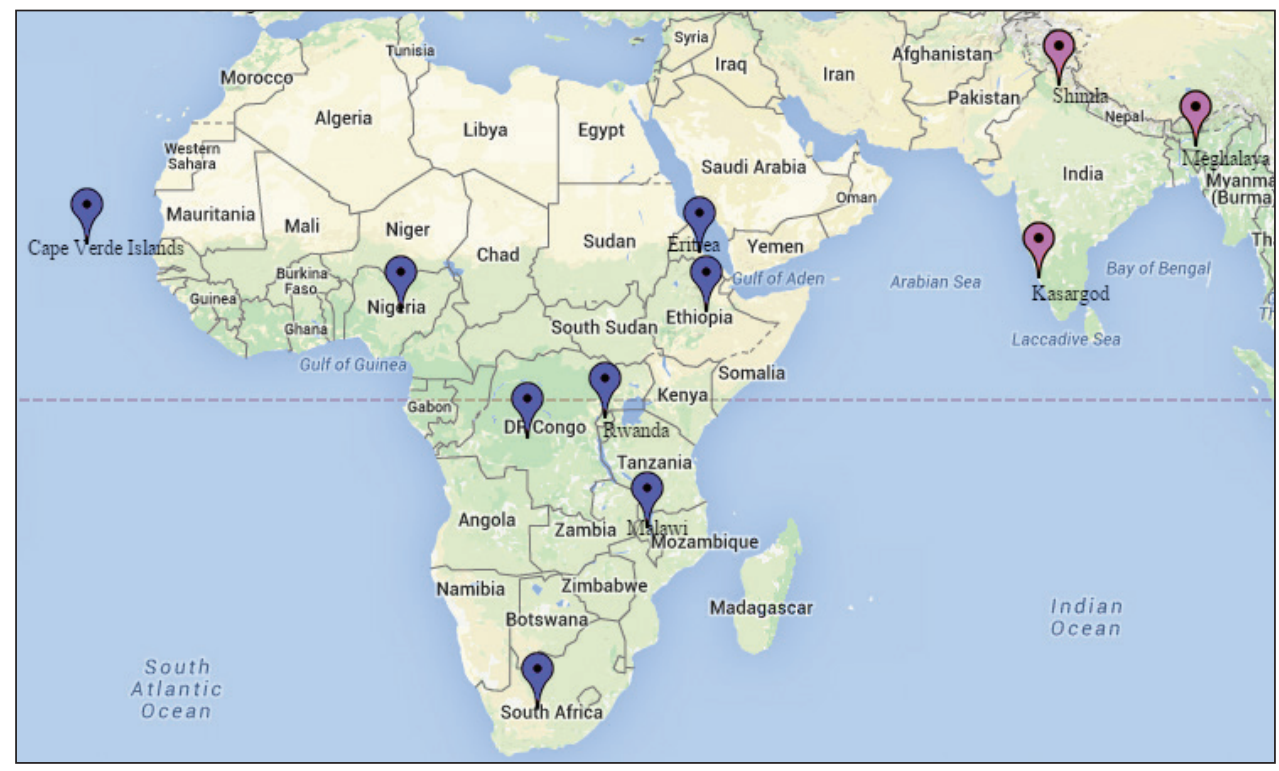

Figure 8. Map showing distribution of Cotesia pistrinariae (blue colored spots in Africa) and Cotesia trabalae (pink colored spots in India).

anterior margin/width at posterior margin: $>1 v s<1$ in both northern populations; (iii) ratio of length of fore wing veins r/2RS: 1.22 vs $0.75-1.06$ in both northern populations; (iv) T3 coloration remains the same as other tergites vs T3 yellowish brown in northern populations (more yellowish in Shimla population); (v) on an average -70 white colored cocoons laid upright on a single host $v s \sim 125$ brown colored cocoons in both northern populations.

The reasons for the colour differences in the cocoons seen is not clear, but it might relate to different conditions (e.g. of humidity) pertaining at the time of their construction. The caterpillar with brown cocoons was collected from Shimla (northern India) which is humid in August while the caterpillar with white colored cocoons was collected in December from southern India (during the dry period).

\section{Acknowledgements}

AG is thankful to the Indian Council of Agricultural Research, New Delhi and to Dr. Abraham Verghese, Director, ICAR-NBAIR, for research support. Specimens collected during the surveys undertaken under the ICAR project "Network Project on Insect Biosystematics" and ICAR-CABI E-UK collaborative project on "The study of biological control of invasive plant species and their natural enemies". 


\section{References}

van Achterberg C, Polaszek A (1996) The parasites of cereal stem borers (Lepidoptera: Cossidae, Crambidae, Noctuidae, Pyralidae) in Africa, belonging to the family Braconidae (Hymenoptera: Ichneumonoidea). Zoologische Verhandelingen 304: 1-123.

Austin AD, Dangerfield PC (1992) Synopsis of Australasian Microgastrinae (Hymenoptera: Braconidae), with a key to genera and description of new taxa. Invertebrate Taxonomy 6(1): 1-76. doi: 10.1071/IT9920001

Fernandez-Triana J, Ward D (2015) Microgastrinae Wasps of the World. http://microgastrinae.myspecies.info [2015/07/02]

Huber JT, Sharkey MJ (1993) Structure. In: Goulet H, Huber JT (Eds) Hymenoptera of the world: an identification guide to families. Agriculture Canada Research Branch, Monograph No. 1894E, Ottawa, Canada, 13-59.

Ivanova NV, deWaard JR, Hebert PDN (2006) An inexpensive, automation-friendly protocol for recovering high-quality DNA. Molecular Ecology Notes 6: 998-1002. doi: $10.1111 / j .1471-8286.2006 .01428 . x$

Kearse M, Moir R, Wilson A, Stones-Havas S, Cheung M, Sturrock S, Buxton S, Cooper A, Markowitz S, Duran C, Thierer T, Ashton B, Mentjies P, Drummond A (2012) Geneious Basic: an integrated and extendable desktop software platform for the organization and analysis of sequence data. Bioinformatics 28(12): 1647-1649. doi: 10.1093/bioinformatics/ bts199

Lanfear R, Calcott B, Ho SYW, Guindon S (2012) PartitionFinder: combined selection of partitioning schemes and substitution models for phylogenetic analyses. Molecular Biology and Evolution 29(6): 1695-1701. doi: 10.1093/molbev/mss020

Mason WRM (1981) The polyphyletic nature of Apanteles Foerster (Hymenoptera: Braconidae): A phylogeny and reclassification of Microgastrinae. Memoirs of the Entomological Society of Canada 115: 1-147. doi: 10.4039/entm113115fv

Ratnasingham S, Hebert PDN (2007) BOLD: The Barcode of Life Data System (www. barcodinglife.org). Molecular Ecology Notes 7: 355-364. doi: 10.1111/j.14718286.2007.01678.x

Ronquist F, Teslenko M, van der Mark P, Ayres DL, Darling A, Hohna S, Larget B, Liu L, Suchard MA, Huelsenbeck JP (2012) MrBayes 3.2: Efficient Bayesian phylogenetic inference and model choice across a large model space. Systematic Biology 61: 539-542. doi: 10.1093/sysbio/sys029

Smith MA, Woodley NE, Janzen DH, Hallwachs W, Hebert PDN (2006) DNA barcodes reveal cryptic host-specificity within the presumed polyphagous members of a genus of parasitoid flies (Diptera: Tachinidae). Proceedings of the National Academy of Sciences 103: 3657-3662. doi: 10.1073/pnas.0511318103

Smith MA, Wood DM, Janzen DH, Hallwachs W, Hebert PDN (2007) DNA barcodes affirm that 16 species of apparently generalist tropical parasitoid flies (Diptera, Tachinidae) are not all generalists. Proceedings of the National Academy of Sciences 104: 4967-4972. doi: 10.1073/pnas.0700050104 
Smith MA, Rodriguez JJ, Whitfield JB, Janzen DH, Hallwachs W, Deans AR, Hebert PDN

(2008) Extreme diversity of tropical parasitoid wasps exposed by iterative integration of natural history, DNA barcoding, morphology and collections. Proceedings of the National Academy of Sciences of the USA 105: 12359-12364. doi: 10.1073/pnas.0805319105

Szépligeti G (1904) Hymenoptera. Fam. Braconidae. Genera Insectorum 22: 1-253.

Whitfield JB (1997) Subfamily Microgastrinae. In: Wharton RA, Marsh PM, Sharkey MJ

(Eds) Manual of the New World genera of the family Braconidae (Hymenoptera). Special

Publication No. 1, International Society of Hymenopterists, Washington, 333-364.

Wilkinson DS (1929) Seven new species of Braconidae. Bulletin of Entomological Research 20: 443-455. doi: 10.1017/S0007485300021416

Yu DSK, van Achterberg C, Horstmann K (2012) Taxapad 2012, Ichneumonoidea 2011. Database on flash-drive, Ottawa, Ontario. http://www.taxapad.com 